\title{
Performance Evaluation of Free Space Optical Wireless Communication
}

\author{
Md. Ebrahim Hossain \\ Lecturer \\ Department of Computer Science and Engineering \\ Leading University, Sylhet 3112, Bangladesh
}

\author{
Taskin Noor Turna \\ Lecturer \\ Department of Information and Communication \\ Engineering \\ Pabna University of Science and Technology, Pabna, \\ Bangladesh
}

\begin{abstract}
Radio on free space optics has become a momentum in research because of its cost effectiveness and efficiency in transferring data at a high rate that is comparable to that for optical fiber media. The transmission data rate is limited in fiber due to dispersion and nonlinearity, such effects do not prevail in FSO communication links. The data rate depends mainly on the switching speed of the optoelectronic devices. With the characteristics of free space being random in nature, the performance of RoFSO is primarily governed by atmospheric conditions. In this report, the performance of the orthogonal frequency division multiplexing (OFDM) signal in free space is evaluated and compared against its counterpart radio frequency (RF) wireless communication systems. Simulations have been done on the atmospheric conditions by means of modeling the scintillation effect using log-normal distribution. The performance of the proposed system under two different baseband modulations, namely OFDM-PSK (phase shift keying) and QAM (quadrature amplitude modulation) in weak turbulence conditions is studied. It is found that PSK performs better than QAM. Also the $M$-ary performance analysis shows that 3-5 dB improvement in the signal to noise ratio is obtained for OFDM based FSO transmission compared to RF based wireless transmission.
\end{abstract}

\section{Keywords}

Optical wireless communication, Radio frequency, Free space optics.

\section{INTRODUCTION}

Wireless optical communications (WOC) becomes a real alternative, since it allows transferring data with high-bandwidth requirements with the additional advantages of wireless systems. Moreover, a wireless optical communication System offers, when compared with RF technology, an intrinsic narrower beam; less power, mass and volume requirements, and the advantage of no regulatory policies for using optical frequencies and bandwidth. Again in satellite communication, optical wireless communication is becoming more important for its own advantages which are mentioned below.

Optical wireless communication, also known as Free Space Optics communications or Free Space Photonics refers to the transmission of modulated visible or infrared beams through the atmosphere to obtain optical communications. Like fiber, Free Space Optics uses lasers to transmit data, but instead of enclosing the data stream in a glass fiber, it is transmitted through the air. Free Space Optics (FSO) works on the same basic principle as Infrared television remote controls, wireless keyboards or wireless Palm devices. Optical wireless can be an extension of $\mathrm{RF}$ and millimeter wave links with very high $(>1 \mathrm{~Gb} / \mathrm{s})$ bandwidth. RF communications in licensed bandwidth can provide higher capacity but spectrum licenses are expensive and
RF in unlicensed band is limited in bandwidth. Some advantages of Optical wireless communication are given below.

1) There are no licensing requirements

2) No tariffs are required for its utilization.

3) There are no radiofrequency (RF) radiation hazards (eyesafe power levels are maintained if required).

4) It has a large bandwidth, which enables very high data rates.

5) It is small, light, and compact.

6) It has low power consumption.

7) Reusability: Enables use of same communication equipments and wavelengths by nearby systems.

8) Cannot be intercepted easily

9) Cannot be interpreted with spectrum analyzers or RF meters.

The major drawback of using optical wireless communication is that here the leasers are used as sources which is a problem for optical wave because it propagates through the turbulent atmosphere. Moreover, fog, rain, snow, haze, and generally any floating particle can cause extinction of the signal-carrying laser beam intensity. In a worst case scenario the intensity attenuation can be strong enough to cause link outages, leading to a high bit error-rate that inevitably decrease the overall system performance and limits the maximum length for the optical link.

The bandwidth of the optical wireless is 10,000 times higher than the highest frequencies used by RF technologies. Furthermore, 1000 independent data channels can be grouped into the air on a single optical beam using wavelength division multiplexing thus providing a potential bandwidth ten million times that of any RF solution

Now a day's interest in optical wireless communication is rapidly growing. Because of the ever growing demand for high-data-rate data transmission as to a large extent current application, such as the high-definition (HD) contents and cloud computing, require great amount of data to be transmitted. It requires more transmission bandwidth. While the fiber-optic infrastructure commonly called network backbone, is capable of coping with current demand, the end user accesses the network data stream through copper based connection and radio-frequency (RF) wireless services, that are inherently slower technologies. As the number of user increases, the radio-frequency spectrum is getting so crowded that there is virtually no room for new wireless services within the RF band. With the added inconvenient of limited bandwidth restriction when using a RF band and the 
license fees that have to be paid in order to use such a band. Regarding cooper-based technologies and the lower-speed connections, compared with the backbone, that are offered such as DS1 (digital line subscriber), cable modems, or T1's (transmission system 1), they are alternatives that makes the service provider to incur in extra installation costs for deploying the wired network through the city.

The simulation of this work is carried out for various values of $M$ (the number of symbols on the carrier) in the $M$-ary modulation. Two different modulations, PSK and QAM, are considered here. As is conventional, the performance of the wireless OFDM communication is inferior to that of the FSO based OFDM. The performances of the QAM base-band modulated FSO-OFDM and the wireless QAM based OFDM systems for $M=2$ are similar to that of PSK modulated FSO OFDM. But QAM fails to provide better performance in the FSO environment for higher values of $M$. It has good performance with $M=2$, but with increase in value of $M$, the BER increases. Another limitation of this work is that only the performance of PSK and QAM is analyzed here. But different types of modulation techniques can be analyzed to get better performance.

\section{SYSTEM MODEL}

The proposed model of the work is described in this chapter.
The considered area of the system, how the system works, OFDM transmitter and receiver system are described in details here.

\subsection{System Model}

In a digital system the transmitter comprises a laser which is modulated to emit pulse and there is lens which makes the beam parallel or aligns the beam carrying the optic signal. Here the atmosphere is considered as the propagation channel. But the problem is when the weather is not normal like when it is foggy or stormy or something like this, the light beams are scattered which degrades communication performance.

Even sometimes in clear atmosphere dust and gas molecule scatters the light beam and degrades communication performance. I want to concentrate on specific issues of fog and haze in the atmospheric channel.

In a typical application, the transmission range is in between 100 to 5000 meter. The transmitter and the receiver are placed on the rooftops of high-rise buildings, on billboards or bridges. The receiver includes the receiver, a detector and a decision making unit. The aperture, whose size determines the total amount of collected power, gathers the light within a set radial distance from the optic axis of transmission.

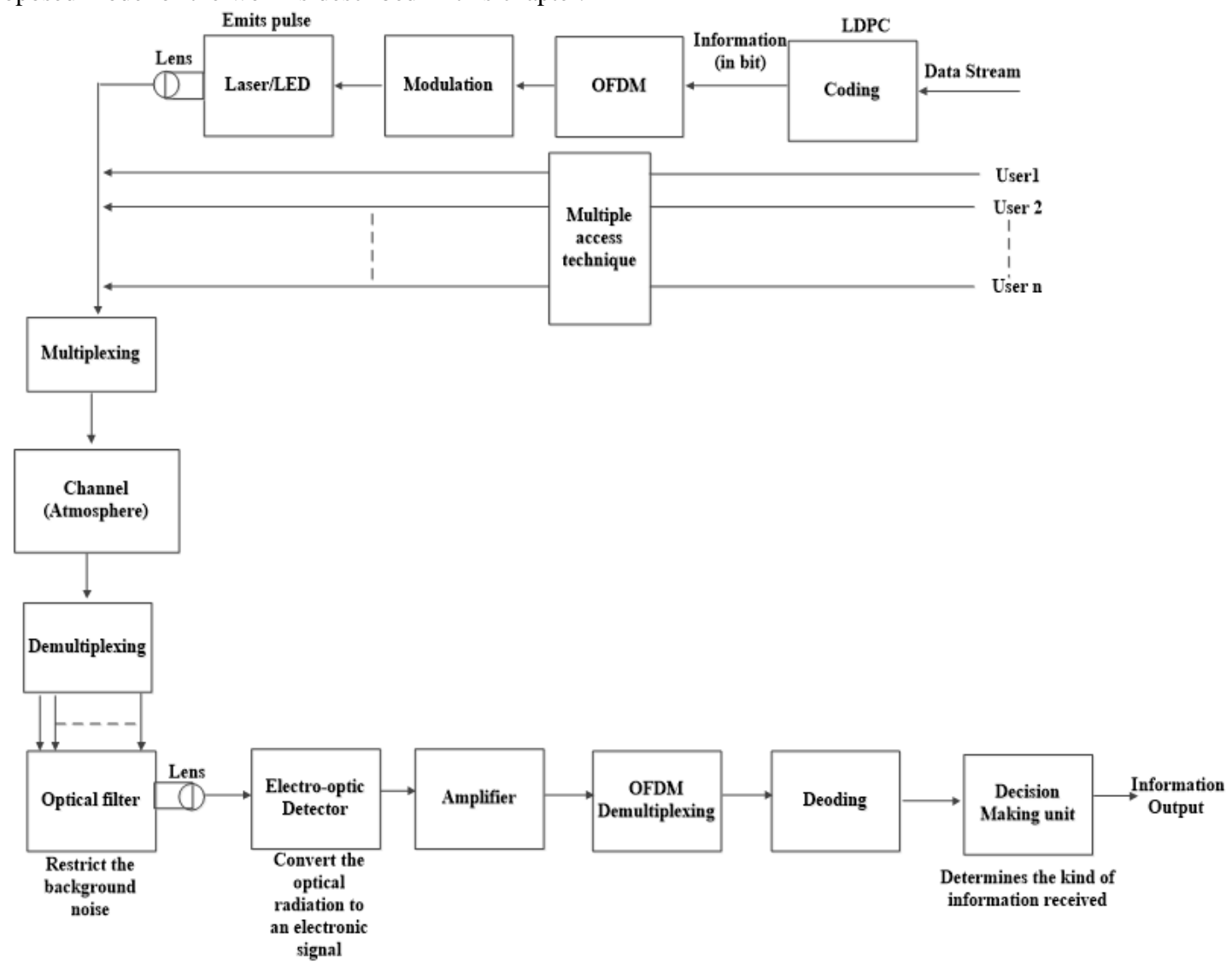

Fig. 1. System model

The technically simplest digital modulation scheme is amplitudeshift keying (2 ASK). In optical systems it is referred to as on-off keying (OOK). OOK is an intensity modulation scheme where the light source (carrier) is turned on to transmit a logic "one" and turned off to transmit a "zero".

Laser or LED can be used to emit pulse. Initially these pulses are not emitted in a sequential manner. A lens is used to make the beam parallel to each other. The beams are then multiplexed before sending through atmosphere. At the receiving end there is a de-multiplexer which de-multiplexed the received beam sequentially. The beams are sent through an optical filter which restricts the background noise. The electro optic detector is the photoelectric element which converts the optical radiation to an electronic signal that is subsequently amplified. The decision making unit decodes the signal and determines the kind of information received based on the signal amplitude and time of interval. 


\subsection{OFDM system model}

OFDM is a multicarrier modulation that divides the high data rate signal into lower data rate signals and transmits them in parallel form. The subcarriers are base-band modulated using any constant envelope modulation technology: PSK, QAM, QPSK etc. In OFDM, the subcarrier spacing is selected in such a way that each subcarrier is orthogonal to each other one. Such subcarriers are generated using the Inverse Fast Fourier Transform (IFFT) at the transmitter, and the Fast Fourier Transform (FFT) is employed at the receiver. This makes the receiver design simple, since just a one-tap or a two-tap equalizer is required [6].

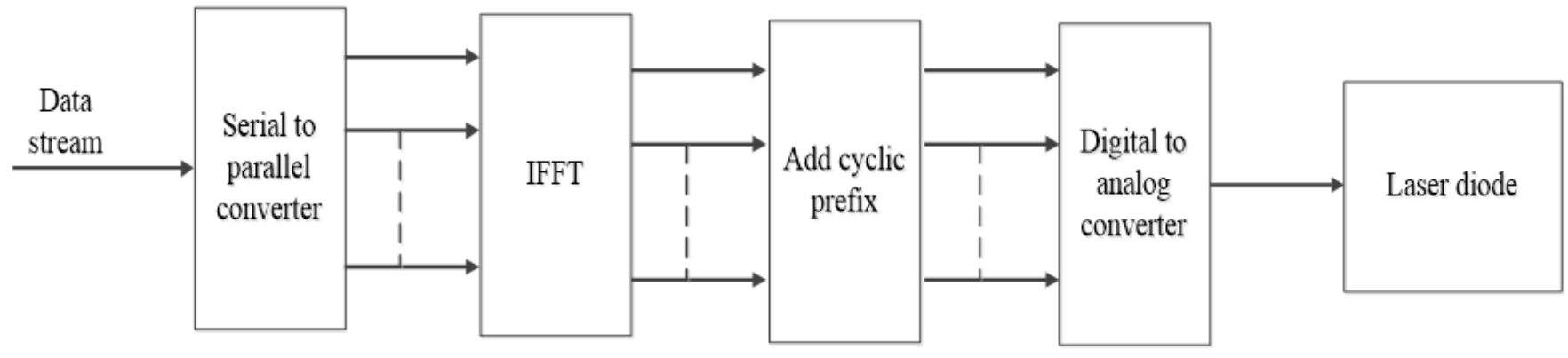

Fig. 2. OFDM transmitter system

The block diagram of the FSO-OFDM transmitter is shown in figure 3.2. At the transmitter, the input signal is taken as a series of bits/symbols which are base-band modulated; this is also called mapping. This converts the signal into the complex form. The mapped signal is converted from serial to parallel form and the IFFT is computed to obtain the OFDM symbol. To the

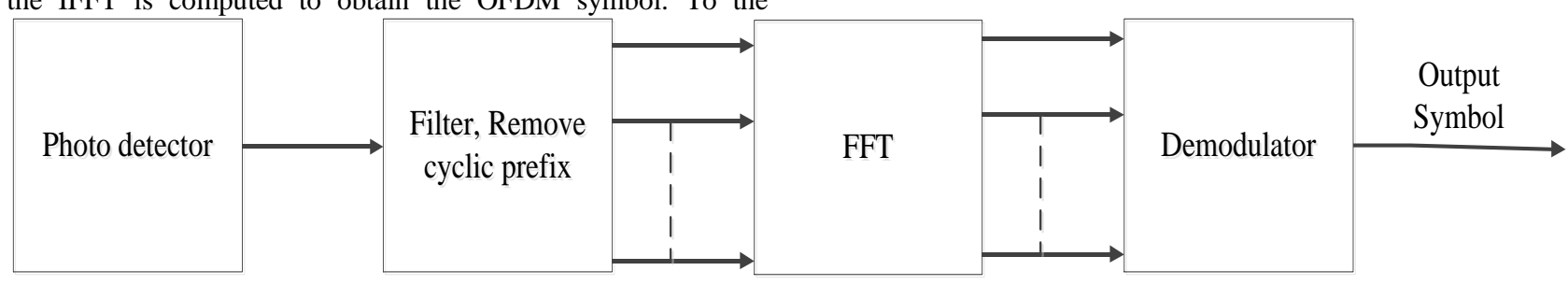
through free space.

Fig. 3. OFDM receiver system

At the receiver, the reverse process is carried out after detection by the photodiode, and the FFT is taken to convert the OFDM symbol back into complex bit sequences. Demapping converts the complex signal into the original bit sequences.

\section{ANALYSIS}

The analysis and the results of the work are described in this chapter. The mathematical model of the system, simulation parameters, performance evaluation is also discussed.

\subsection{Mathematical model}

The Complex OFDM sequence is represented as

$S(t)=\frac{1}{\sqrt{T}} \sum_{0}^{K-1} a_{k} e^{\frac{j 2 \pi k t}{T}} w(n T)$

Where $\mathrm{T}$ is the symbol duration, the $a_{k}$ are the data bits, and $\mathrm{w}(\cdot)$ is the rectangular window function.

The OFDM signal with $\mathrm{N}$ subcarriers modulates the intensity of the laser diode and it is considered to be linear;

$$
P(t)=P_{t} \sum_{n=0}^{N-1} m_{n} s_{n}(t)
$$

Where $P_{t}$ is the average transmitted optical power, $m_{n}$ is the optical modulation index (OMI) for each subcarrier and the total OMI is given as

$$
m_{\text {total }}=\frac{1}{N} \sqrt{\sum_{n=0}^{N-1} m_{n}^{2}}
$$

For an FSO link, the field at any point can be written as the product of the free space attenuation and stochastic amplitude, generated OFDM symbol, cyclic prefix bits/guard bands are added for improved system performance, and this is followed by parallel to serial conversion and digital to analog conversion. This OFDM signal modulates the laser diode and is then transmitted

to describe the field variation. At the receiver, the input to the photodiode is given as

$$
P_{r x-F S O}(t)=P(t) L_{a t t n} L_{s c i n t} X+n_{F S O}(t)
$$

Where $P(t)$ is power output from the laser. $L_{a t t n}$ is the loss due to the atmosphere (including rain and attenuation loss), $L_{\text {scint }}$ is the loss due to atmospheric turbulence and $X$ is the signal fading due to atmospheric turbulence effects.

Assuming that FSO link noise can be filtered out, the output current of the photodiode is given as

$$
\begin{gathered}
I(t, X)=I_{p h} P(t)+n_{o p t}(t) \\
I_{p h}=\rho L_{\alpha} L_{s c i n t} L_{a t t n} P_{t} X
\end{gathered}
$$

Where $L_{\alpha}$ is the FSO pointing loss and geometrical loss, $P(\mathrm{t})$ is the average transmitted power, $I_{p h}$ is the dc component of the received photocurrent $I(t, X), \rho$ is the responsivity of the photodiode and $n_{\text {opt }}$ is the arbitrary waveform generator noise. This Gaussian noise model includes the thermal noise, the shot noise, and the relative intensity noise (RIN) processes. The expression for the noise power is thus written as

$$
N_{0}=\frac{4 T_{a b} k_{B} F}{R_{L}}+2 q I_{p h}+(R I N) I_{p h}^{2}
$$

Where $k_{B}$ is Boltzmann's constant, $T_{a b}$ is the absolute 
temperature, $F$ is the noise figure of the receiver, $R_{L}$ is the photo detector load resistance, RIN is the relative intensity noise and $q$ is the electron charge.

Attenuation depends on wavelength atmospheric attenuation. Atmospheric transmission, $\mathrm{T}$ is described by Beer's law $(\mathrm{A}=$ bcd)

$$
T\left(L_{12}\right)=e^{-\alpha_{e} L_{12}}
$$

Here $\alpha_{e}$, is the atmospheric extinction coefficient. $\mathrm{V}_{\mathrm{m}}=$ Visibility

$$
\begin{gathered}
T\left(V_{m}\right)=e^{-\alpha_{e} v_{m}}=0.05 \\
\text { i.e., }-\alpha_{e} v_{m}=\ln (0.05) \\
\text { i.e., } \alpha_{e} v_{m}=-\ln (0.05) \\
\text { i.e., } \alpha_{e}=-\frac{\ln (0.05)}{v_{m}} \\
\text { i.e., } \alpha_{e}=\frac{3}{v_{m}}\left[\mathrm{Km}^{-1}\right]
\end{gathered}
$$

The main phenomenon influencing $\alpha_{\mathrm{e}}$ in atmospheric optical communication windows is the mie scattering on particles. Its wavelength dependency can be taken into account by modifying $\alpha_{\mathrm{e}}$ with a $\lambda$ selective term so that it finally reads

$$
\alpha_{e, p a r t} \approx \frac{3}{v m}\left(\frac{\lambda}{550}\right)^{-0.5853 \sqrt{v m}}\left[K^{-1}\right]
$$

(At the wavelength of 550nm, Human eye has the highest sensitivity)

$$
e^{-\alpha_{e, p a r t} L_{12}}=10^{-\frac{\alpha_{1, p a r t}}{10} L_{12}}
$$

The atmospheric attenuation is the $\mathrm{dB}$ scale.

$$
\begin{gathered}
e^{-\alpha_{e, \text { part }}} L_{12}=10^{-\frac{\alpha_{1, \text { part }}}{10}} L_{12} \\
\text { i.e., }-\alpha_{e, \text { part }}+\ln \left(L_{12}\right)=\ln \left(10^{-\frac{\alpha_{1, \text { part }}}{10}} L_{12}\right) \\
\text { i.e., }-\alpha_{\text {e,part }}+\ln \left(L_{12}\right)=\ln \left(10^{-\frac{\alpha_{1, \text { part }}}{10}}\right)+\ln \left(L_{12}\right) \\
\text { i.e., }-\alpha_{e, \text { part }}=\ln \left(10^{-\frac{\alpha_{1, \text { part }}}{10}}\right) \\
\text { i.e., }-\alpha_{e, \text { part }}=-0.23 \alpha_{1, \text { part }} \\
\text { i.e., } \alpha_{e, \text { part }}=0.23 \alpha_{1, \text { part }}
\end{gathered}
$$

Here, $\alpha_{e, p a r t}=1 / \mathrm{km}$

Specific attenuation $\alpha_{1, \text { part }}=\mathrm{dB} / \mathrm{km}$

\subsection{Atmospheric turbulence and beam interruptions}

It is possible to evaluate turbulence extent with the help of the structural parameter of the index of refraction.

The relative optical variance at $\mathrm{R}_{\mathrm{XA}}$ is

$$
\sigma_{I, r e l}^{2}=\mathrm{KC}_{\mathrm{n}}{ }^{2}(2 \pi / \lambda)^{7 / 6} \mathrm{~L}_{12}{ }^{11 / 6}
$$

Here $\mathrm{K}$ is the constant which value is 1.23 for plane wave and

\begin{tabular}{|c|c|c|}
\hline Symbol & Description & Value \\
\hline $\mathrm{L}$ & $\begin{array}{l}\text { Distance between } \\
\text { the transmitter and } \\
\text { the receiver }\end{array}$ & $1000 \mathrm{~m}$ \\
\hline $\mathrm{R}$ & $\begin{array}{l}\text { Photo detector } \\
\text { responsivity }\end{array}$ & $0.8 \mathrm{AW}^{-1}$ \\
\hline Q & Electron Charge & $1.6^{*} 10^{-19} \mathrm{C}$ \\
\hline $\mathrm{K}_{\mathrm{b}}$ & Boltzman's constant & $3.374 * 10^{-23}$ \\
\hline $\mathrm{T}$ & Temperature & $300(\mathrm{~K})$ \\
\hline $\mathrm{R}_{\mathrm{L}}$ & Load Resistance & $50 \Omega$ \\
\hline RIN & $\begin{array}{c}\text { Relative intensity } \\
\text { noise }\end{array}$ & $-150 \mathrm{~dB}$ \\
\hline $\mathrm{B}$ & Filter bandwidth & $6(\mathrm{MHz})$ \\
\hline $\mathrm{W}_{0}$ & $\begin{array}{l}\text { Input beam spot } \\
\text { radius }\end{array}$ & $20 \mathrm{~mm}$ \\
\hline $\mathrm{F}_{0}$ & $\begin{array}{l}\text { Input radius of } \\
\text { curvature }\end{array}$ & $-16 \mathrm{~m}$ \\
\hline $\mathrm{N}$ & $\begin{array}{l}\text { Number of } \\
\text { subcarriers }\end{array}$ & 52 \\
\hline $\mathrm{T}$ & Symbol duration & $4 \mu \mathrm{s}$ \\
\hline
\end{tabular}
0.5 for spherical wave.

When the relative variance of the optical intensity at $\mathrm{R}_{\mathrm{XA}}$ is less than 1 that is considered as a weekly turbulent atmosphere.

Then,

$$
\alpha_{\text {turb }} \approx\left|10 \log \left(1-\sqrt{\sigma_{I, \text { nel }}^{2}}\right)\right|(16)
$$

\subsection{Measuring BER:}

$$
B E R=\frac{n_{e}}{N_{B}}
$$

$n_{e}$ Indicates the number of the received error bits and $\mathrm{N}_{\mathrm{B}}$ is the number of transmitted bits.

\section{SIMULATION RESULTS AND DISCUSSIONS}

Parameters associated with free space and the numerical values used for the simulation are given in TABLE 1.

\section{TABLE 1: FSO OFDM LINK PARAMETERS}

The stream of data is superimposed on the subcarriers to generate the required OFDM symbol. A cyclic prefix is added and all symbols are made serial to form a train for the OFDM symbol. Assuming the RF conversion to be perfectly done and this block to be linear, the base band OFDM signal is used to optically modulate the laser diode (LD). The light from the LD is transmitted in free space, which is assumed to be lognormally distributed. At the receiver, the received signal is expected to perform the reverse processes at the transmitter. In some of the literature, a point optical source is considered to have a plane or spherical wave pattern, while analyses are also carried out with collimated Gaussian beam waves. The plane wave propagation model is suitable for space-ground transmission and the Gaussian beam model gives a good approximation for terrestrial transmission.

The aperture averaged scintillation index for varying distance and wavelength has been evaluated. Distance is varied over a range of $0-2 \mathrm{~km}$ with four different wavelengths, namely 780 , 980,1330 and $1550 \mathrm{~nm}$. It is observed that irrespective of the wavelength, for short distances in the range 500-800 m, the scintillation fluctuates from 0.5 to 0.9 . As the wavelength is increased from 780 to $1550 \mathrm{~nm}$, the curve shifts both to the right and upward. This shows that for longer wavelength the fluctuation is greater compared to that for shorter wavelength. 
Thus it is preferred to use shorter wavelength for free space communication, over longer wavelength. Hence for terrestrial applications, a laser diode with a Gaussian beam is used with a shorter wavelength.

During the generation of the OFDM signal at the transmitter, the input data are base-band modulated and then the IFFT is taken to generate the OFDM symbol. At this stage we analyze the performance of $M$-ary PSK modulation in the RF wireless environment and the FSO environment. Also the received signal power is generated by an algorithm given by Epple [6]. The simulation is carried out for various values of $M$ in the $M$ ary modulation. Two different modulations, PSK and QAM, are considered here. The result shows that the performance of PSK is better than that of QAM. As is conventional, the performance of the wireless OFDM communication is inferior to that of the FSO based OFDM.

Fig. 4 shows that at low SNR the performances of the FSOOFDM system and the wireless OFDM system are almost the same. The deviation in the performance starts from $0 \mathrm{~dB}$, where the performance of the FSO-OFDM is improved over the wireless OFDM performance. There is an improvement of $2-4 \mathrm{~dB}$ for $M=2-8$. But at the same time as the number of symbols on the carrier increases, the BER also increases. As more symbols are packed onto the same carrier, the probability of making errors increases, even at positive values of the SNR. In order to achieve a low BER we would like to select binary PSK, as compared to other $M$-ary PSK modulations.

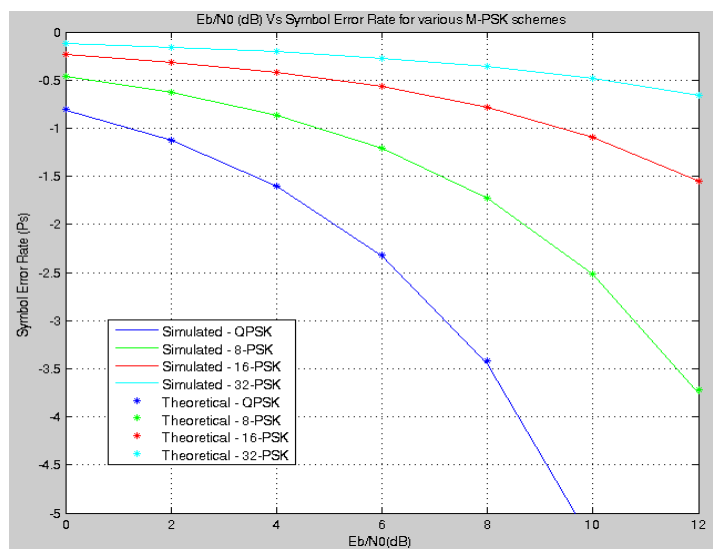

Fig. 4. BER vs SNR for PSK modulation

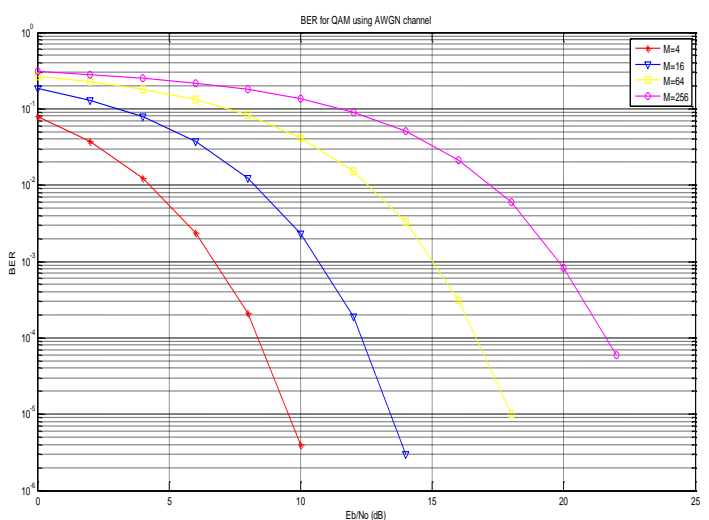

Fig. 5. BER vs SNR for QAM modulationAs shown in Fig. 5 , the performances of the QAM base-band modulated FSO-OFDM and the wireless QAM based OFDM systems for $M=2$ are similar to that of PSK modulated FSO OFDM. But QAM fails to provide better performance in the FSO environment for higher values of $M$. It has good performance with $M=2$, but with increase in value of $M$, the BER increases.

\section{CONCLUSION}

Free space optics (FSO) provides a low cost, rapidly deployable method of gaining access to the fiber optic backbone. FSO technology not only delivers fiber-quality connections, it provides the lowest cost transmission capacity in the broadband industry. As a truly protocol-independent broadband channel, FSO systems complement legacy network investments and work in harmony with any protocol, saving substantial up-front capital investments. An FSO link can be procured and installed for as little as one-tenth of the cost of laying fiber cable, and about half as much as comparable microwave/RF wireless systems. By transmitting data through the atmosphere, FSO systems dispense with the substantial costs of digging up sidewalks to install a fiber link. Unlike RF wireless technologies, FSO eliminates the need to obtain costly spectrum licenses or meet further regulatory requirements. Simulation of the OFDM based free space optical environment using a log-normal distribution shows that the performance of OFDM is improved in free space, as compared to that of a wireless system. Of the two types of modulation used in the simulation environment, we would prefer to use PSK modulation. The order of modulation is also chosen to be 2 rather than a higher order for the $M$-ary modulation technique.

\section{REFERENCES}

[1] Jing Li and M. Uysal, "Achievable information rate for outdoor free space optical communication with intensity modulation and direct detection", GLOBECOM '03. IEEE Global Telecommunications Conference (IEEE Cat. No.03CH37489). Available: 10.1109/glocom.2003.1258717 [Accessed 29 May 2019].

[2] M. Uysal, Jing Li and Meng Yu, "Error rate performance analysis of coded free-space optical links over gammagamma atmospheric turbulence channels", IEEE Transactions on Wireless Communications, vol. 5, no. 6 , pp. 1229-1233, 2006. Available: 10.1109/twc.2006.1638639 [Accessed 29 May 2019].

[3] V. Annovazzi-Lodi, G. Aromataris, M. Benedetti and S. Merlo, "Secure Chaotic Transmission on a Free-Space Optics Data Link", IEEE Journal of Quantum Electronics, vol. 44, no. 11, pp. 1089-1095, 2008. Available: 10.1109/jqe.2008.2001929 [Accessed 29 May 2019].

[4] A. Islam and S. Majumder, "Performance analysis of a free space optical link in the presence of pointing errors with space diversity", 2014 International Conference on Electrical Engineering and Information \& Communication Technology, 2014. Available: 10.1109/iceeict.2014.6919176 [Accessed 29 May 2019].

[5] R. Nee and R. Prasad, OFDM for wireless multimedia communications. Boston: Artech House, 2000.

[6] B. Epple, "Simplified Channel Model for Simulation of Free-Space Optical Communications", Journal of Optical Communications and Networking, vol. 2, no. 5, p. 293, 2010. Available: 10.1364/jocn.2.000293 [Accessed 29 May 2019]. 